\title{
Is early childhood care and education a good investment for Egypt? Estimates of educational impacts, costs, and benefits [Arabic]
}

Caroline Krafft

Follow this and additional works at: https://knowledgecommons.popcouncil.org/departments_sbsr-pgy

Part of the Demography, Population, and Ecology Commons, Disability and Equity in Education Commons, Education Policy Commons, Family, Life Course, and Society Commons, Inequality and Stratification Commons, International Public Health Commons, and the Social Policy Commons How does access to this work benefit you? Let us know!

\section{Recommended Citation}

Krafft, Caroline. 2012. "Is early childhood care and education a good investment for Egypt? Estimates of educational impacts, costs, and benefits [Arabic]," Survey of Young People in Egypt Policy Brief no. 3. Cairo: Population Council. 


\section{مسـح النشء والشباب في مصر ملخص السياسـات}

ملخـص السـياسـات رقهم

\section{التربية والتعليم

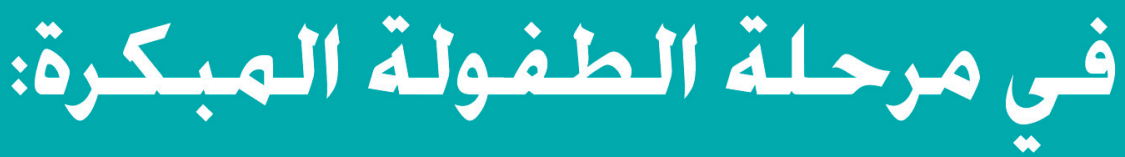

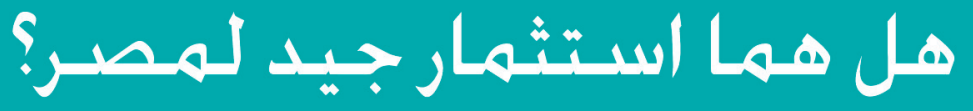

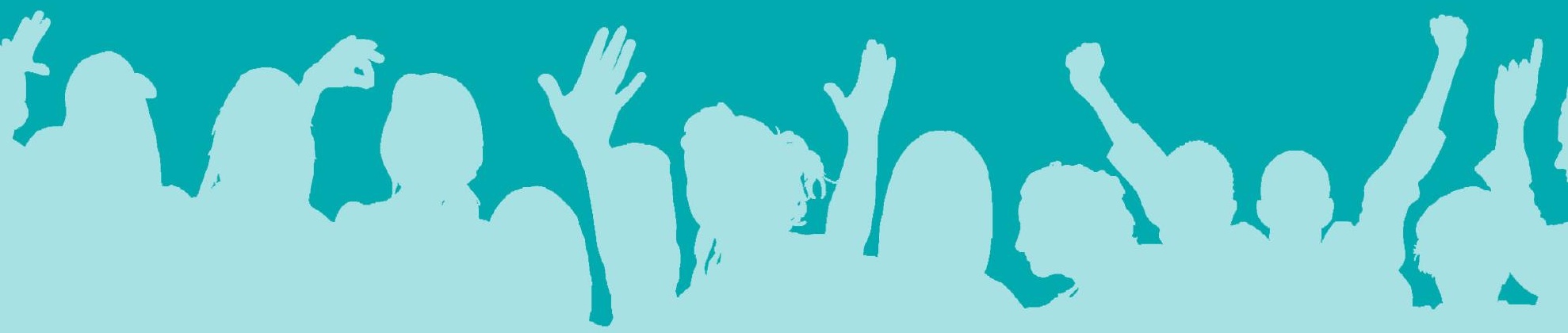

كارولين كر افت

(2) Population Council
FORDFOUNDATION Working with Visionaries on the Frontlines of Social Change Worldwide 


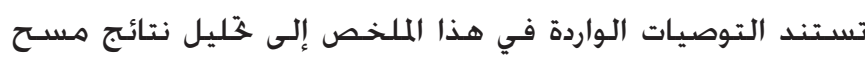

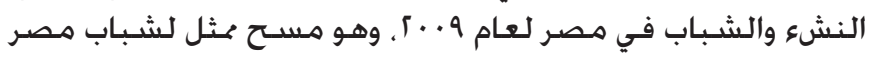

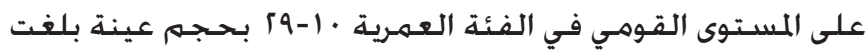

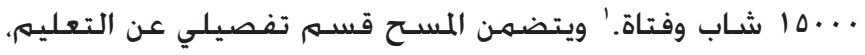

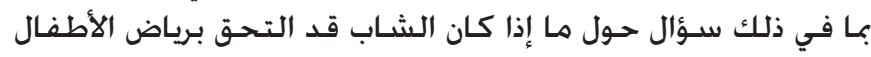

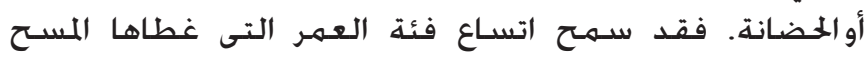

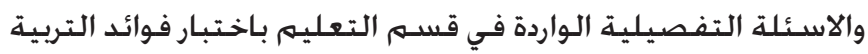
والتعليهم المبكر على افراد المستح.

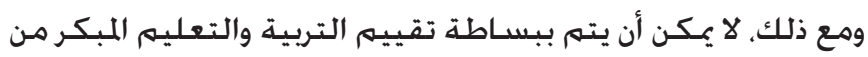

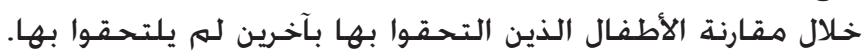

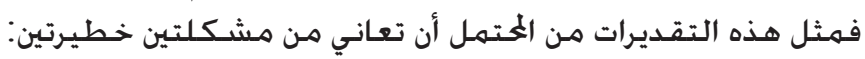

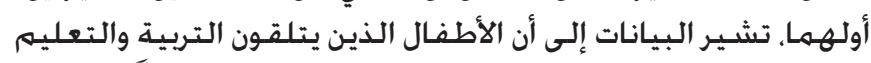

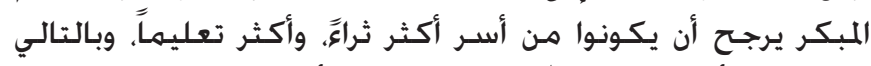

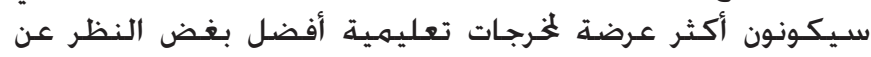

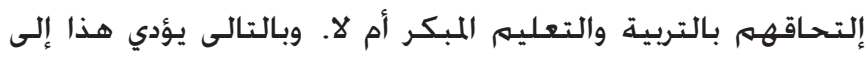

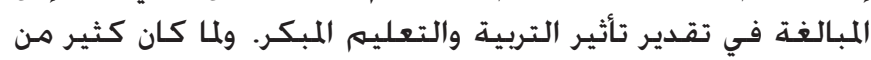

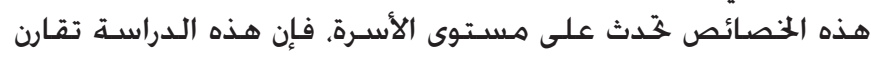

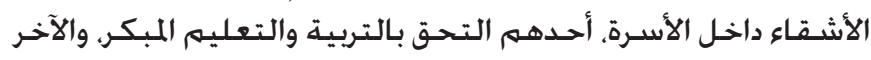

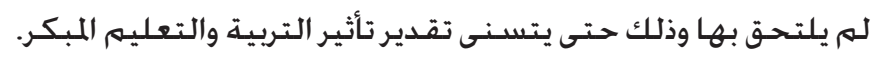

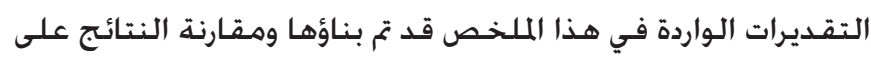

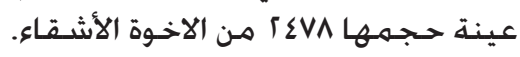

وثاني هاتين المشـكلتين هي أن الكثير من الشـباب الذين شـملهمه

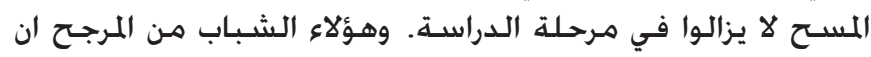

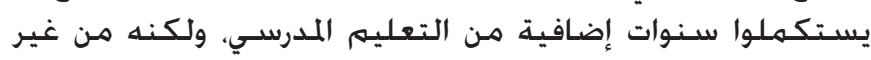

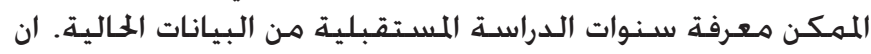

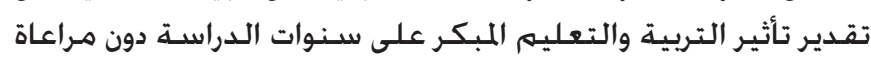

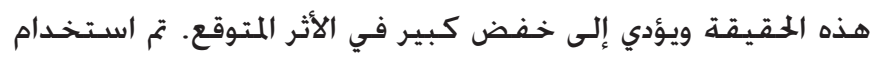

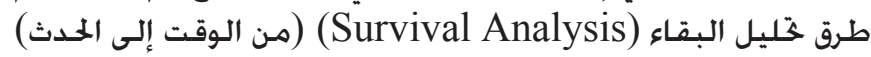

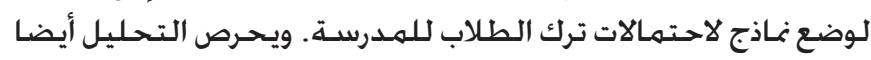

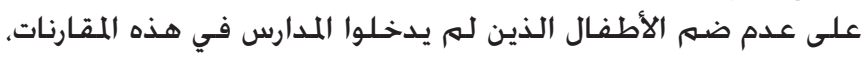

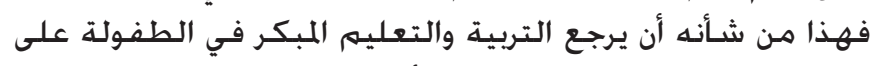
نحو غير صحيح للنية في تعليه الأطفال فقط. لتط.

\section{التربية والتعليهم في مرحلة الطفولة المبكرة}

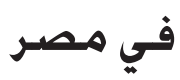

تتخذ التربية والتعليه في الطفولة المبكرة في مصر شكلين

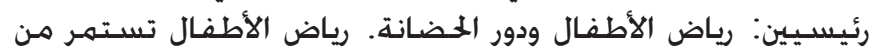

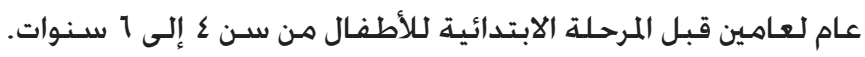

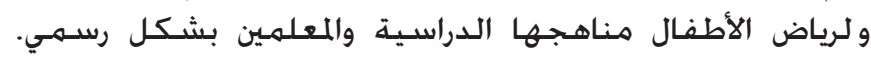

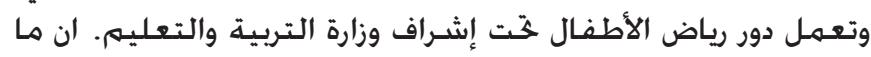

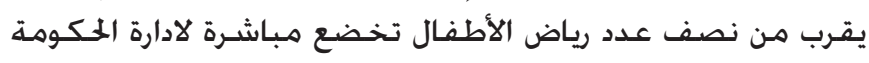

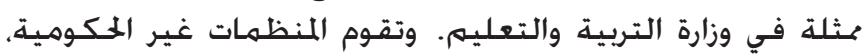
والمدارس الدينية، وأصحاب الأعمال، أو الأفراد بإدارة النسبة المبة المتبقية المكية

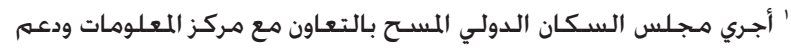

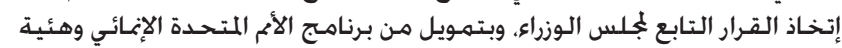

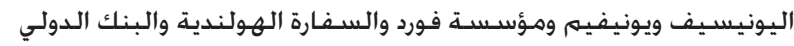
والوكالة الكندية للتنمية الدولية والوكالة السـويدية للتنمية الدولية.
التربية والتعليم في المراحل العمر الاولى التى لتوفي

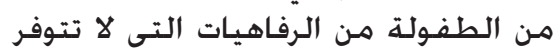

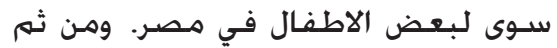

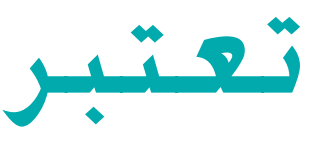

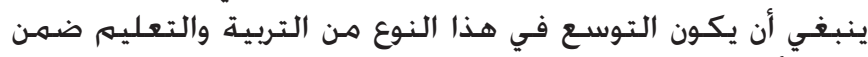

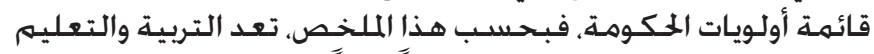

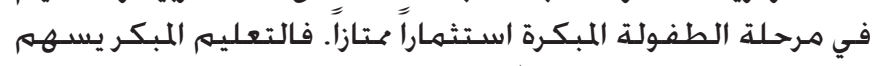

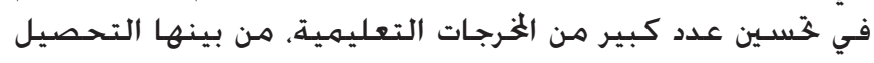

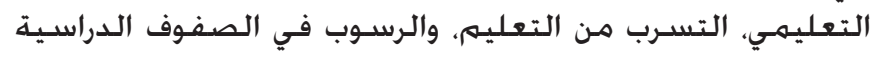

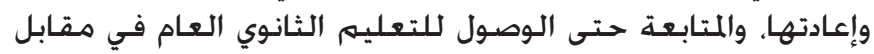

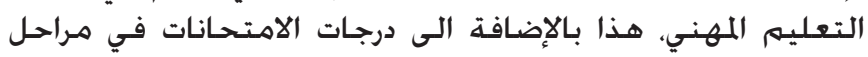

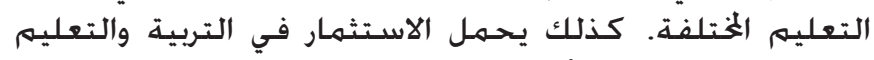

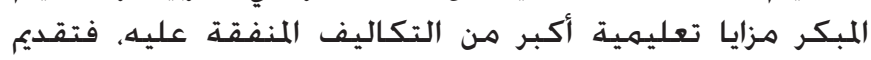

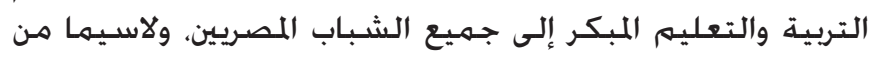

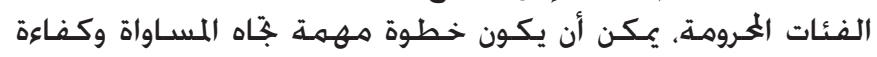
التحصيل في النظام التعليمي.

\section{ما سبب أهمية التربية والتعليم في مرحلة الطفولة المبكرة؛}

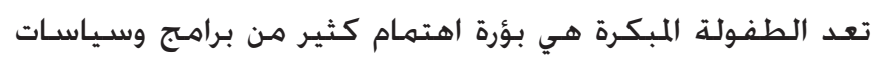

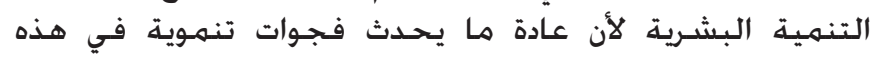

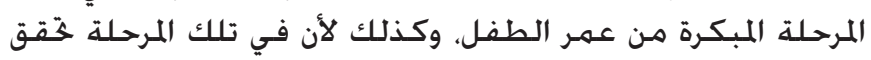

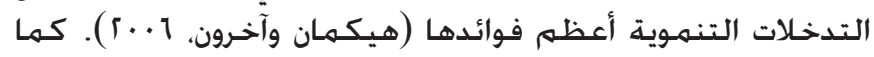

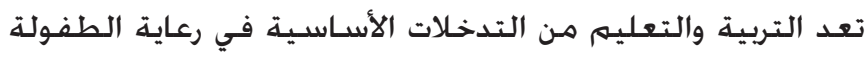

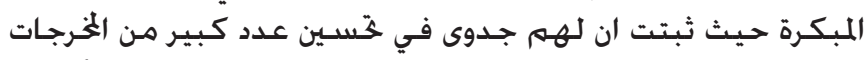

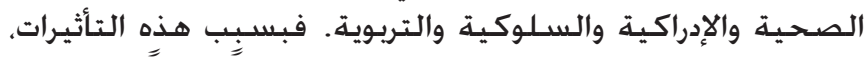

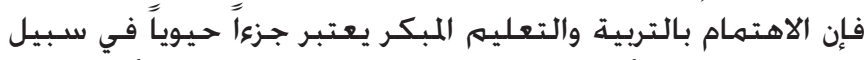

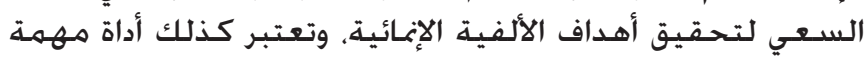

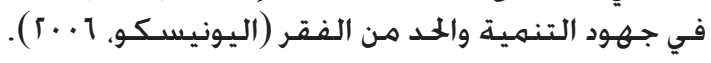

ومما هو جدير بالذكر ان التربية بالإضافة للتعليه في مرحلة إيجات

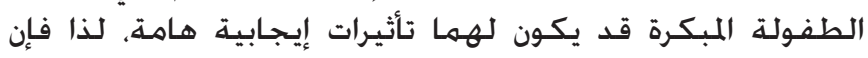

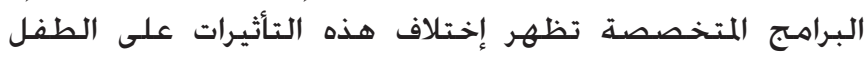

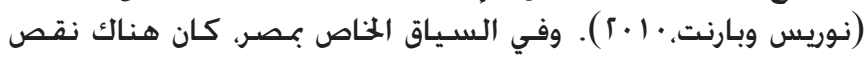

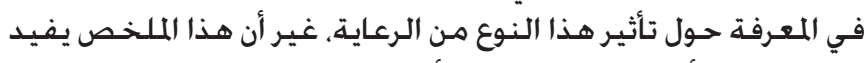

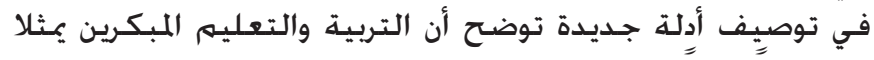

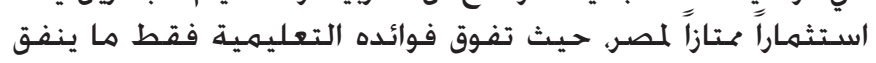
عليهما من تكاليف.

\section{الرسـائل الأسـاسـية}

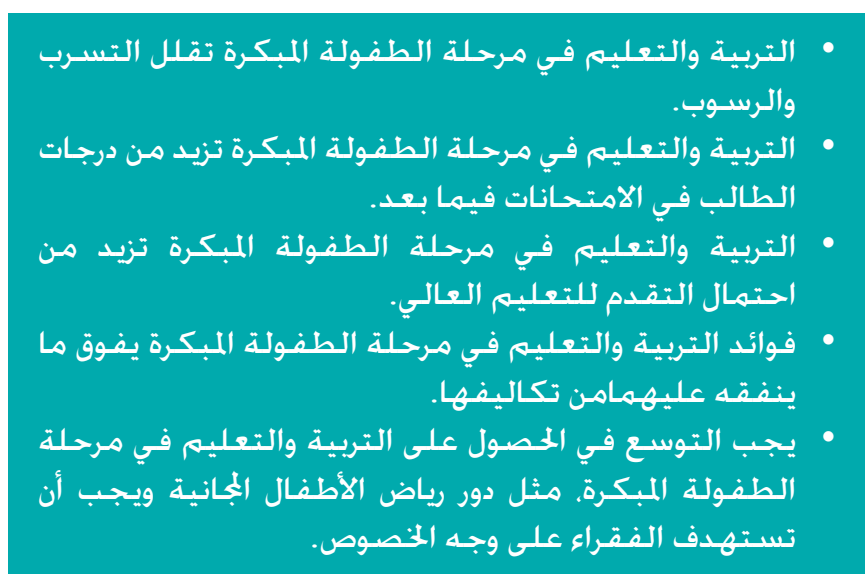


شـكل ؟: نسبة الشباب الباقين في المدرسـة حسب إلتحاقهم بـالتربية

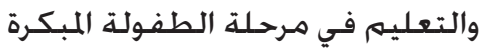

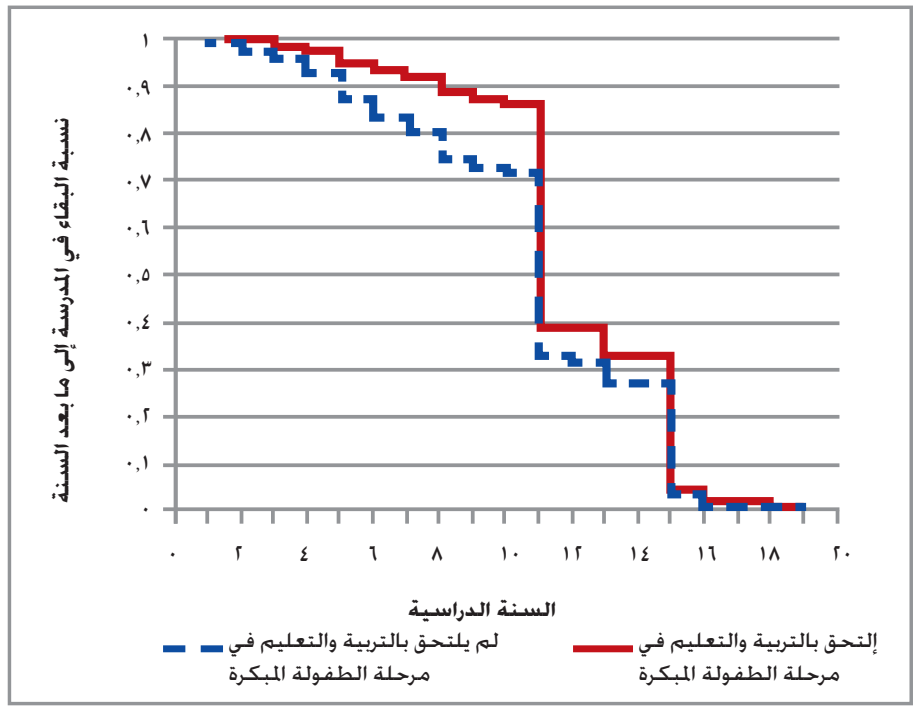

وتشير النتائج الى وجـود إختلاف معنـوى كبير بين من إلتحقـوا بالتربية

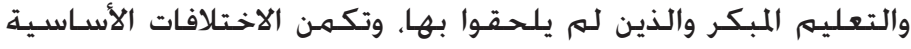

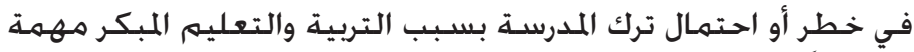

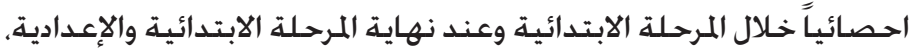

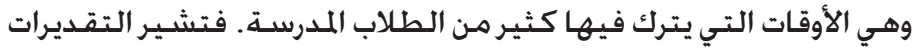

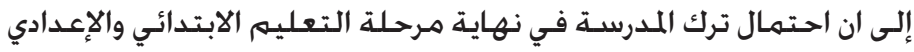

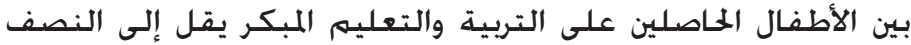

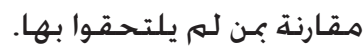

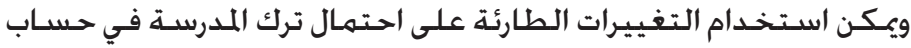

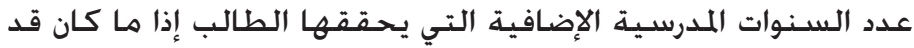

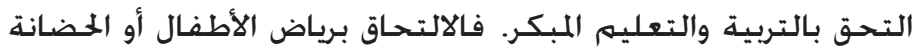

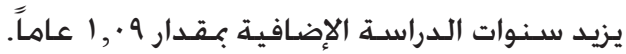

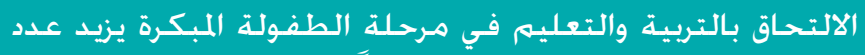

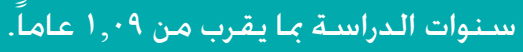

هذه التغييرات في الالتحاق بالمدارس التي من شـأنها أن يكون لها تأثير

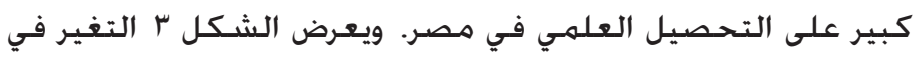

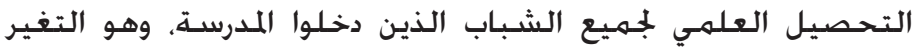

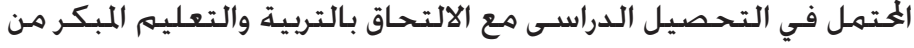

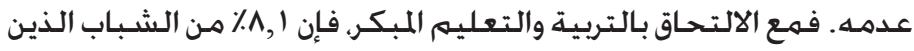

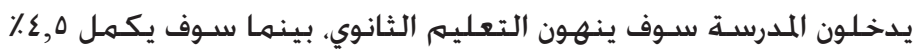

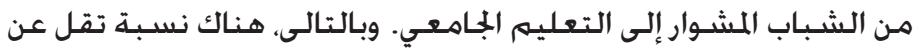

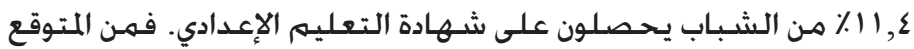

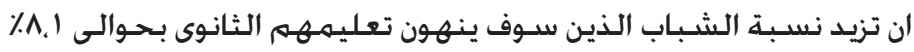

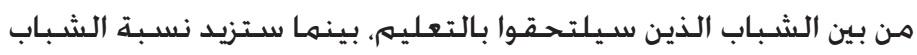

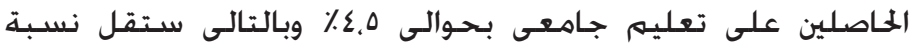

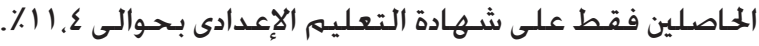

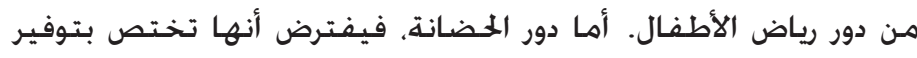

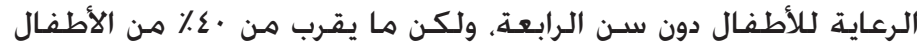

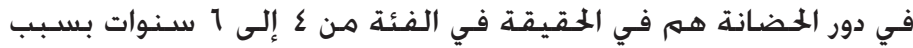

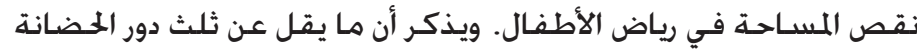

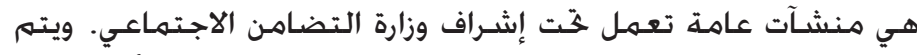

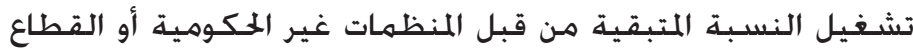

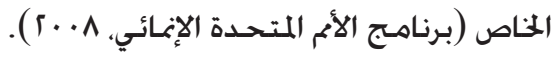
شـكل ا : نسبـة الإلتحاق بالتربية والتعليهم في مرحلة الطفولة المبكرة حسب النوع وسـنة الميلاد

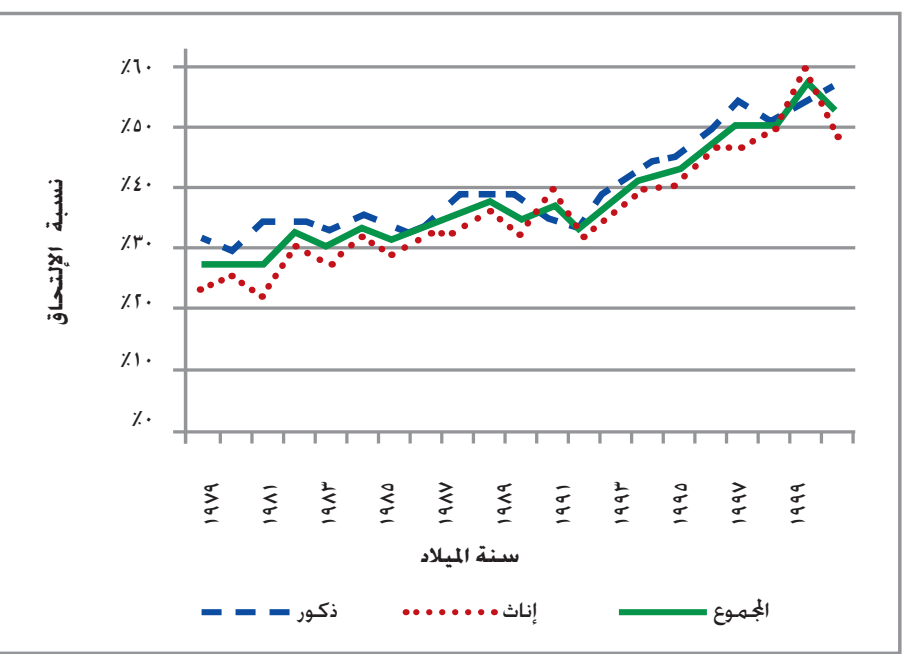

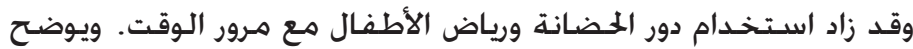

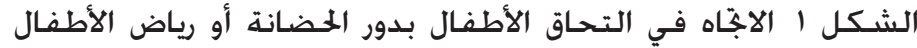

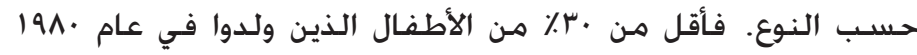

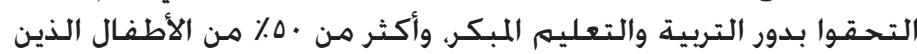

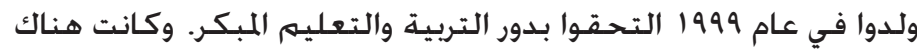

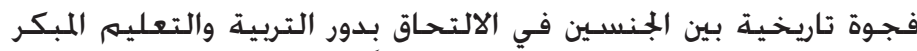

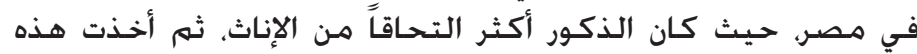

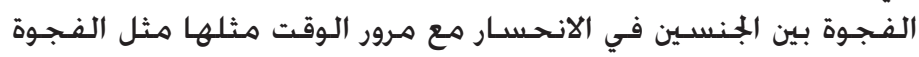

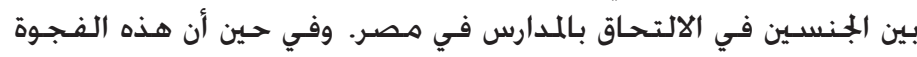

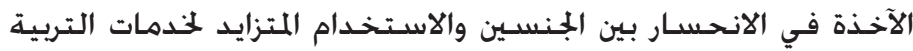

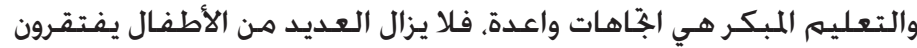

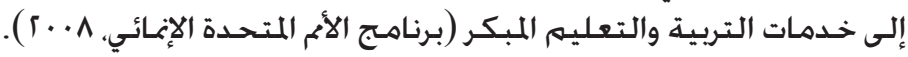

\section{التربية والتعليهم في مرحلة الطفولة المبكرة تسسـن مخرجـات العملية التعليمية فئة}

تؤُثر خـدمات التربية والتعليهم في مرحلة الطفولة المبكرة على عدد كبير

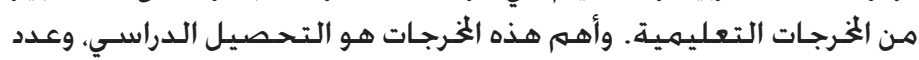

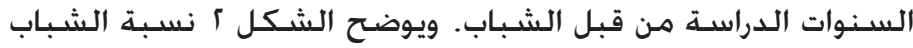

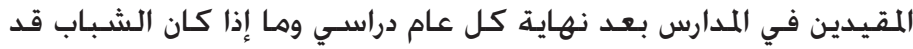

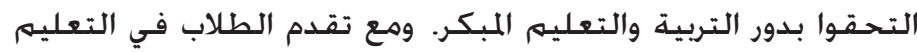

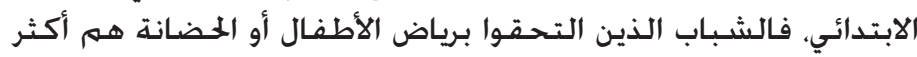

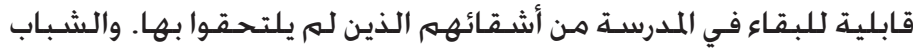

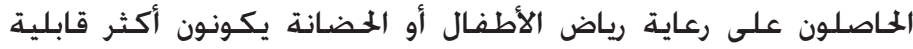

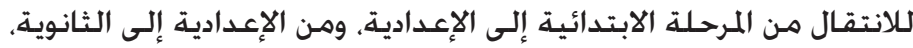
ثم من الثانوية إلى التعليهم العـالي. 


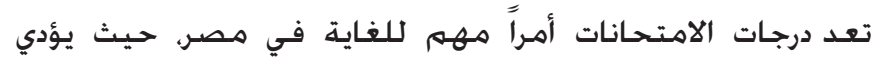

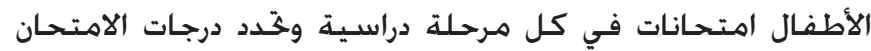

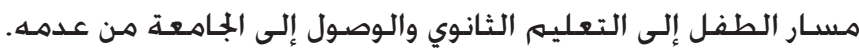

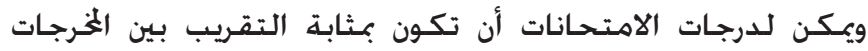

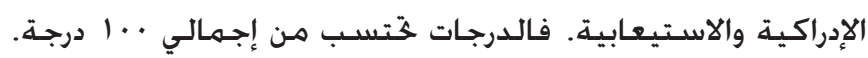

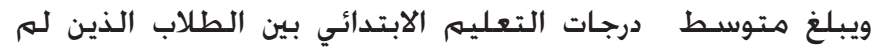

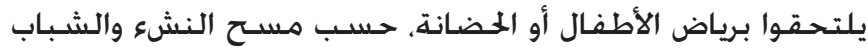

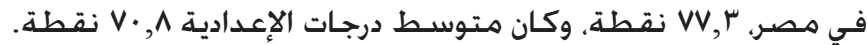

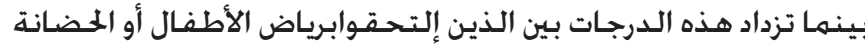

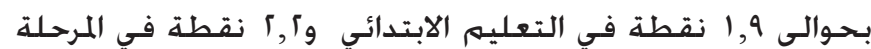
الإعدادية وهو مقدار ضئيل وغير مؤثر.

ويشير التأثير الكبير للتربية والتعليم المبكر على كثير من المخرجات

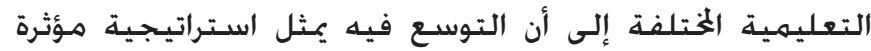

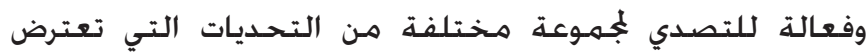

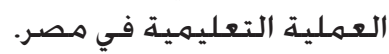

\section{فوائد التربية والتعليم في مرحلة الطفولة المبكرة تتجاوز تكاليفها}

بينما تتضح فوائد التربية والتعليم المبكر في خسسين مخرجات

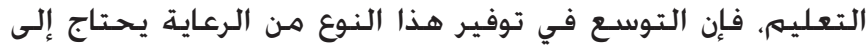

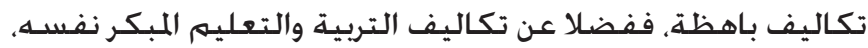

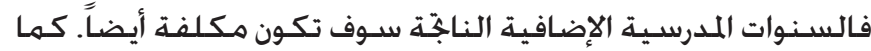

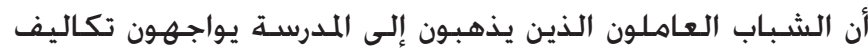

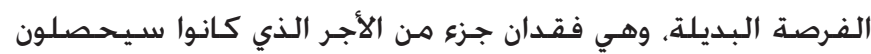

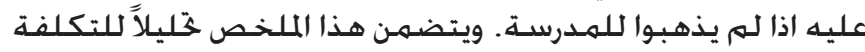

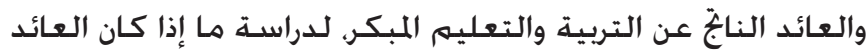
من التربية والتعليه المبكر تفوق تكاليفها.

نسبة العائد إلى التكلفـة للتربية والتعليهم في مرحلة الطفـولة

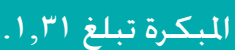

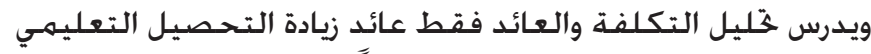

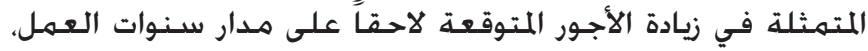

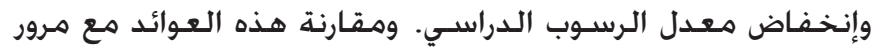

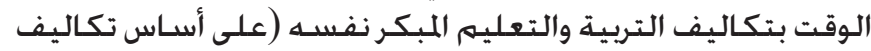

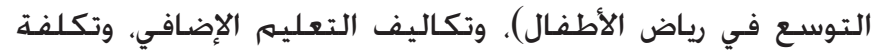

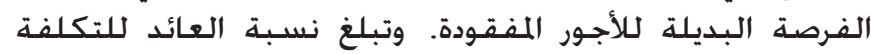

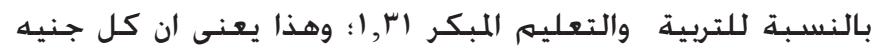

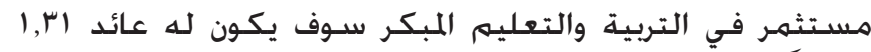

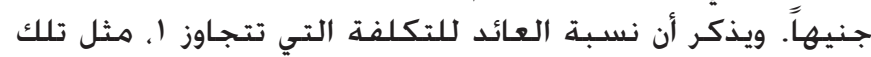

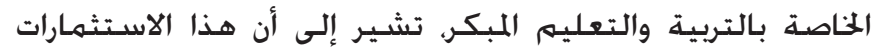

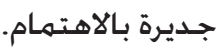

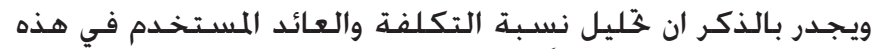

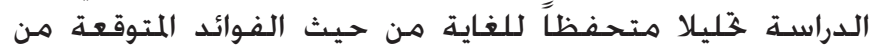

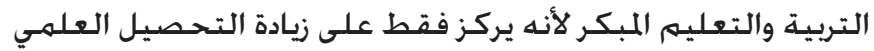

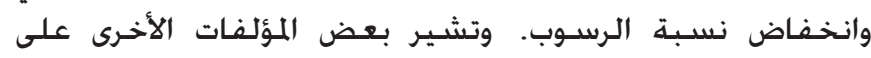

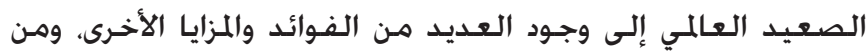

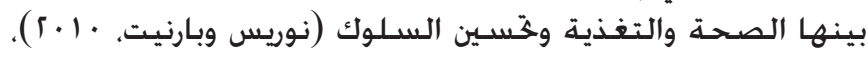

شكل ץ: التغير المحتمل في التحصيل الدراسي مع الإلتحاق بالتربية

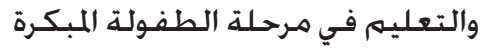

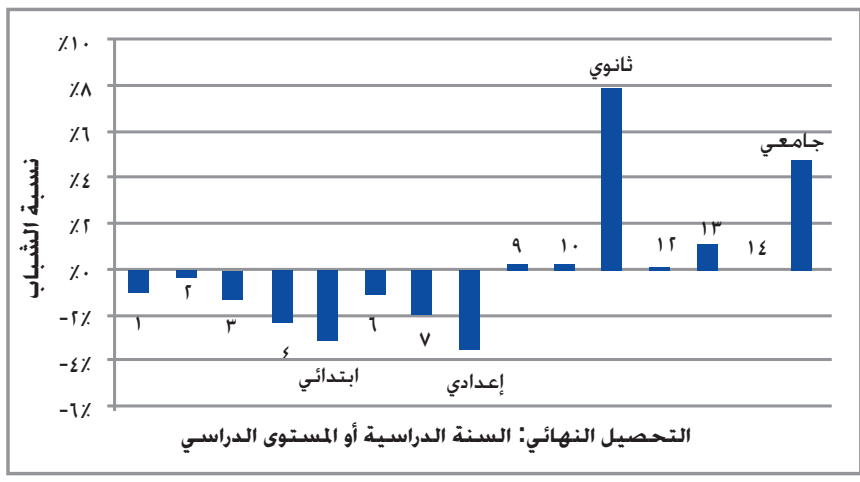

ان زيادة التحصيل التعليمي والانخفاض النسبي في التسرب ليسيا

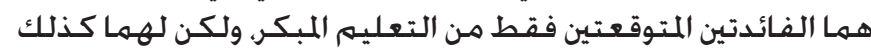

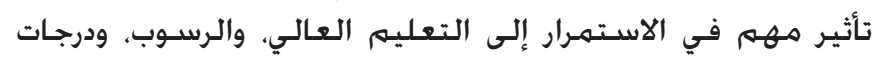

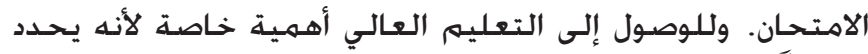

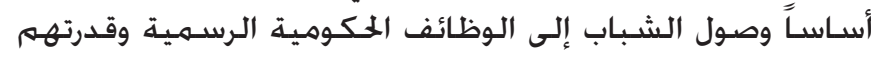

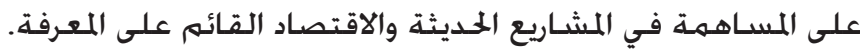

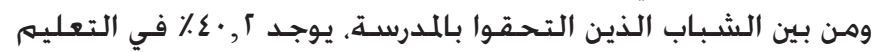

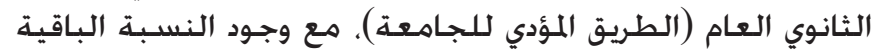

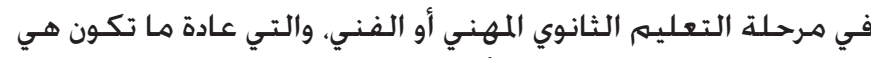

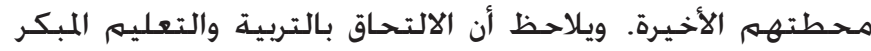

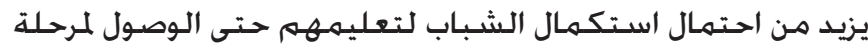

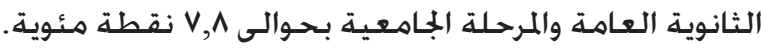
شـكل ع: إعادة السـوات الدراسية بحسب الإلتحاق بالتربية والتعليم

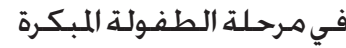

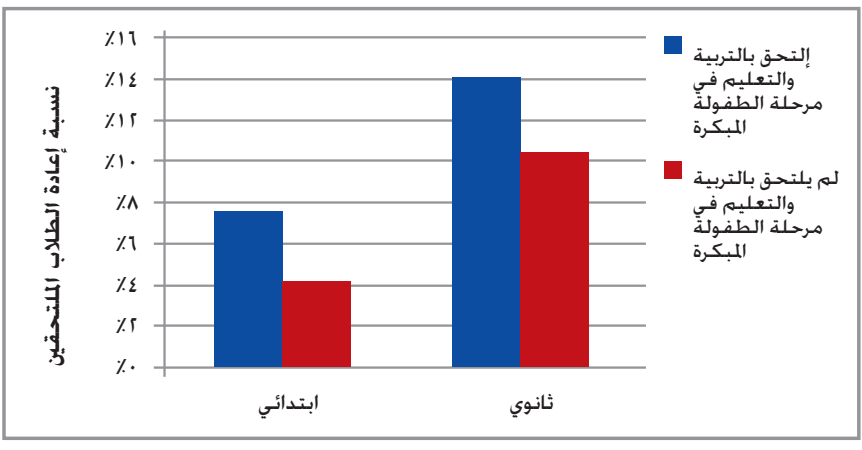

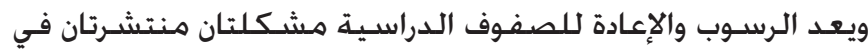

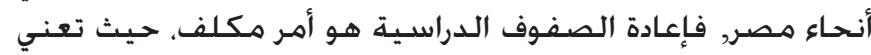

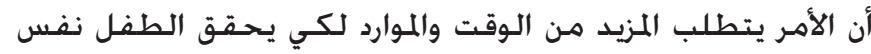

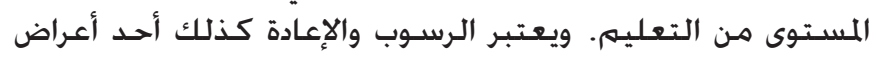

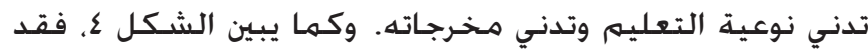

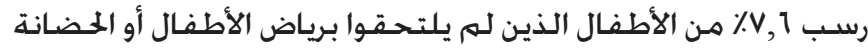

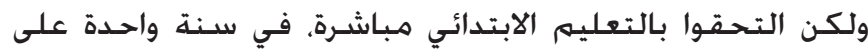

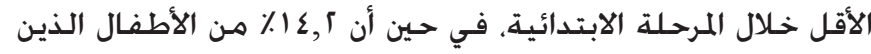

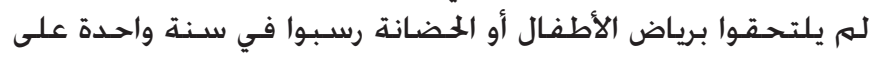

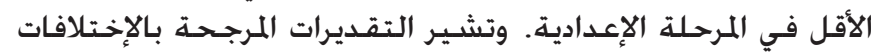

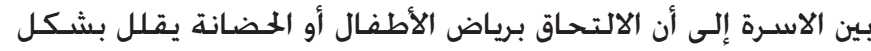

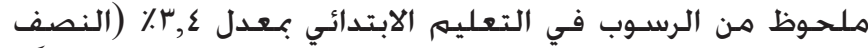

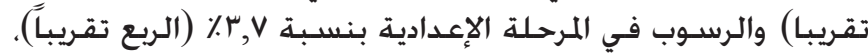

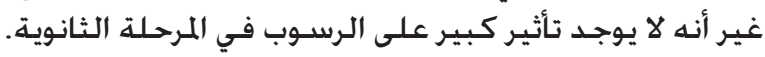


مقارنة بنظرائهم الأكثر ثراء، وذلك بسـبب فقرهم وعدم قدرتهمه

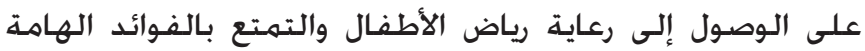

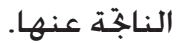

وتعد التكلفـة هي العائق الرئيسي أمام الالتحاق بدور التربية

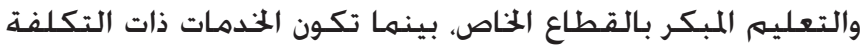

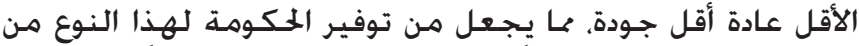

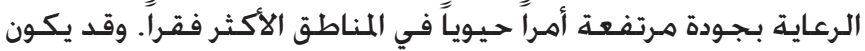

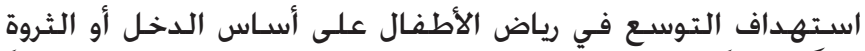

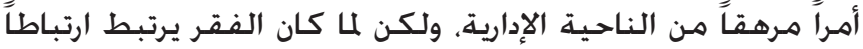

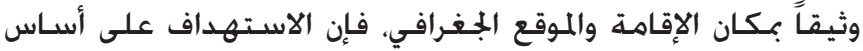

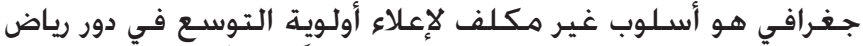

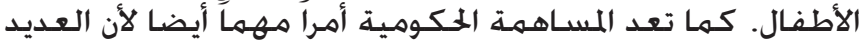
من الفوائد المتوقعة من التربية والتعليهم المبكر إنما هي فوائد عامة المها.

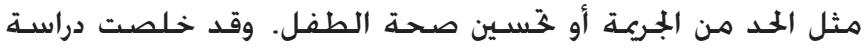

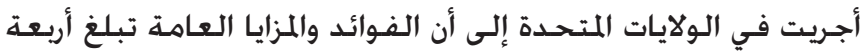

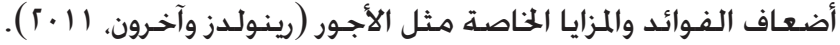
وأوضح هذه التحليل أن التربية والتعليهم المبكر بإمكانهـما تقديم مسـاهمات هامة لنجاح التعليم وفرص الحيح الحياة للشبباب المصري.

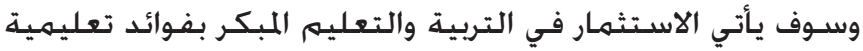

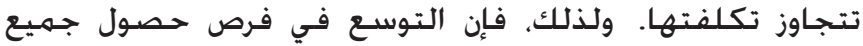

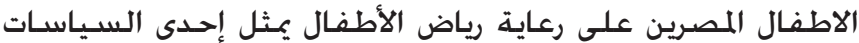

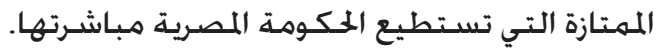

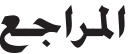

Assaad, R., and C. Krafft. 2010. The Social Background and Attitudes of Higher Education Students and Graduates in Egypt. Report prepared for the Social Science Research Council Project on University Governance and Autonomy in the Changing Landscape of Higher Education in the Arab World. December 2010 (mimeo).

Heckman et al. 2006. "Skill formation and the economics of investing in disadvantaged children," Science. 312: 1900-1902.

Krafft, C. 2011. "Is Early Childhood Care and Education a good investment for Egypt? Estimates of educational impacts, costs, and benefits." Unpublished master's thesis.

Nores, M., and W. S. Barnett. 2010. "Benefits of early childhood interventions across the world: (Under) Investing in the very young," Economics of Education Review. 29: 271-282.

Reynolds, A., J. Temple, B. White, and S.-R. Ou. 2011. "Age 26 cost-benefit analysis of the childparent center early education program, Child Development. 82(1): 379-404.

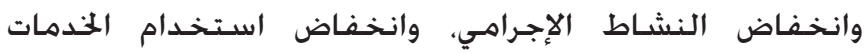

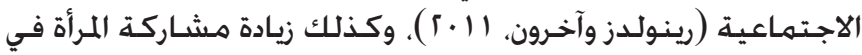

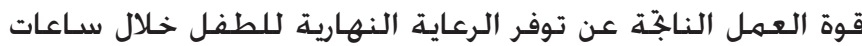

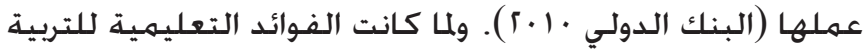

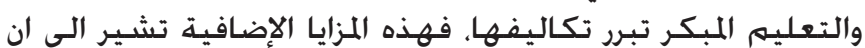
التربية والتعليم في مرحلة الطفولة المبكرة استثـمارا بالغ الأهمية.

\section{ينبغى التوسـع في التربية والتعليهم في مرحلة الطفولة المبكرة}

تقترب مصر من المعدل العالمي للالتحاق بالتعليه الابتدائي.

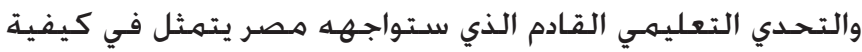

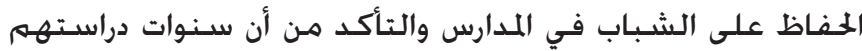

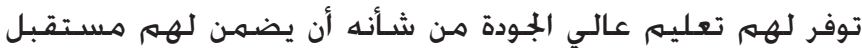

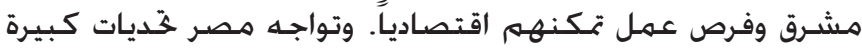

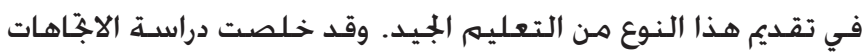

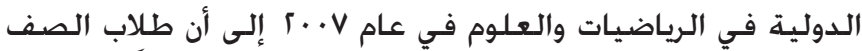

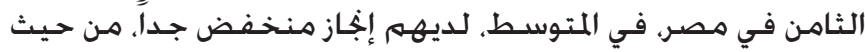

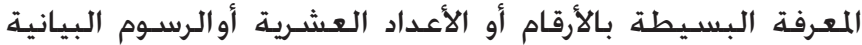

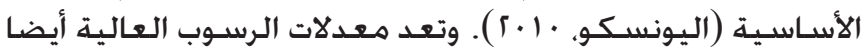

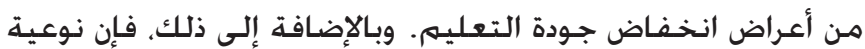

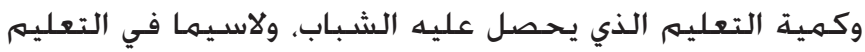

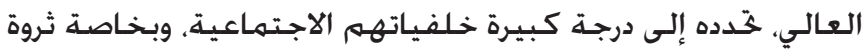

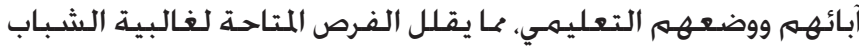

$$
\text { المصري (أسعد وكرافت · ( • ). }
$$

وقد أظهر هذا التحليل أن التربية والتعليم في مرحلة الطفولة

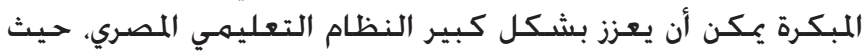

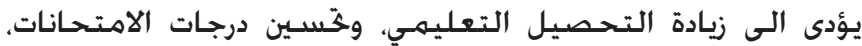

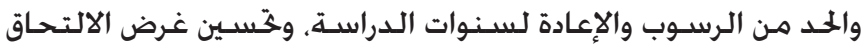

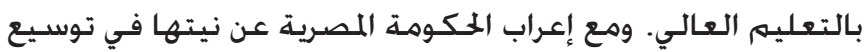

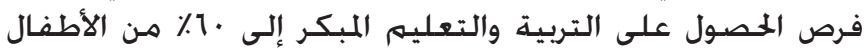

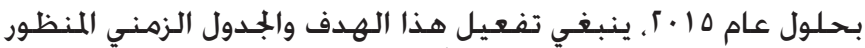

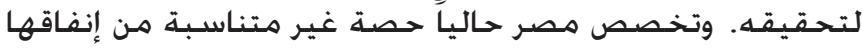

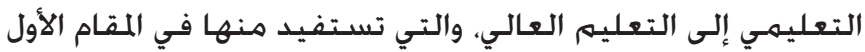

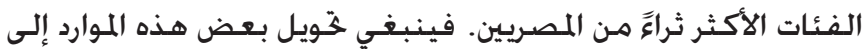

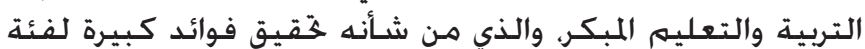

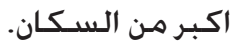

ينبغي أن تتوسـع الحكـومة على وجهـ السـرعة في توفير التربية

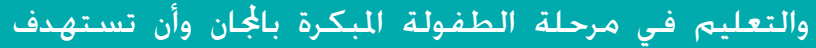
الأطفال في المناطق العحروهة.

وتعد زيادة تقديم الحكومة لبرامج الحضانة ورياض الأطفال الجمانية من ملية

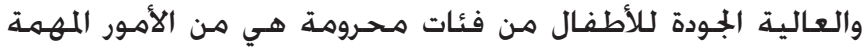

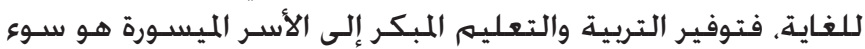

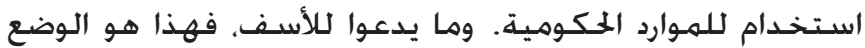

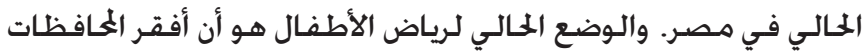

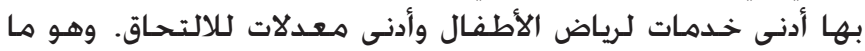

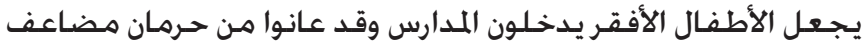


Salehi-Isfahani, D., I. Tulani, and R. Assaad. 2009. "A comparative study of returns to education of urban men in Egypt, Iran, and Turkey." Middle East Development Journal. 1(2): 145-187.

UNDP. 2008. Egypt Human Development Report. UNDP.

UNESCO. 2006. EFA Global Monitoring Report: Strong foundations: Early childhood care and education. Paris: UNESCO.

UNESCO. 2010. EFA Global Monitoring Report: Reaching the Marginalized. Paris: UNESCO.

World Bank. 2010. Turkey: Expanding Opportunities for the Next Generation. Washington, DC: World Bank.

The Population Council confronts critical health and development issues-from stopping the spread of HIV to improving reproductive health and ensuring that young people lead full and productive lives. Through biomedical, social science, and public health research in 50 countries, we work with our partners to deliver solutions that lead to more effective policies, programs, and technologies that improve lives around the world. Established in 1952 and headquartered in New York, the .Council is a nongovernmental, nonprofit organization governed by an international board of trustees 